\section{Fotobiomodulación para la mucositis oral en pacientes oncológicos}

\section{Photobiomodulation for oral mucositis in cancer patients}

\section{Sr. Editor.}

La terapia de fotobiomodulación (PBM-T) consiste en una terapia de luz que usa láseres con el objetivo de reparar tejidos, disminuir la inflamación y el dolor. Cuando la luz es absorbida por el organismo, la energía que tiene la luz se convierte en energía celular, dando inicio a un conjunto de eventos metabólicos, que inducen analgesia, regeneración, o efecto antinflamatorio. Tradicionalmente este tratamiento se ha usado para tratar lesiones deportivas, dolor neuropático, artritis, dolor de cuello o espalda. De forma novedosa en los estudios científicos de los últimos años se ha propuesto el uso de PBM-T como una alternativa prometedora para el manejo y tratamiento de la mucositis oral $(\mathrm{OM})$ en pacientes oncológicos ${ }^{1}$.

En el estudio de Guedes et al. ${ }^{2}$ realizado en 2018 se compararon dos dosis de energía láser administradas a la mucosa oral de pacientes bajo tratamiento oncológico, buscando diferencias en el control de la mucositis, así como en la frecuencia de recurrencias tumorales. Cincuenta y ocho pacientes sometidos a radioterapia fueron asignados al azar en dos grupos, distinguidos según la energía suministrada por la irradiación con láser, a saber, $0,25 \mathrm{~J}$ y 1,0 J. La fotobiomodulación se aplicó cuatro días a la semana con una duración de 10-15 minutos; desde el primer hasta el último día de radioterapia o hasta la resolución de la mucositis. La OM fue significativamente menos frecuente en pacientes que recibieron 1,0 $\mathrm{J}$ de energía, pero los grupos no difirieron con respecto a la gravedad o duración de la OM. La recurrencia tumoral tampoco varió significativamente entre los grupos. La fotobiomodulación con una dosis de energía más alta $(1,0 \mathrm{~J}$ versus $0,25 \mathrm{~J})$ se asocia con un mejor control de la $\mathrm{OM}$ inducida por radioterapia y no aumenta significativamente el riesgo de recurrencia neoplásica.

En el estudio de Pires Marques et al. ${ }^{3}$ realizado en 2020 se evaluó la eficacia de PBM-T, solo o combinado con terapia fotodinámica (PDT), para el tratamiento de la $\mathrm{OM}$ en pacientes con cáncer. Se reclutaron 56 pacientes y se dividieron en dos grupos ( $\mathrm{n}=28$ cada uno): PBM-T y PBM-T + PDT. En el grupo PBM-T, se aplicó láser de bajo nivel a 61 puntos en la cavidad oral, una vez por semana durante 4 semanas (longitud de onda $660 \mathrm{~nm}$, potencia $100 \mathrm{~mW}$, densidad de energía $142 \mathrm{~J} / \mathrm{cm}^{2}$, energía puntual 4J, tiempo de irradiación 40 s) En el grupo PBM-T + PDT, además de PBM-T como se describió anteriormente, los pacientes se enjuagaron con $20 \mathrm{ml}$ de enjuague bucal fotosensibilizante (curcumina $1,5 \mathrm{~g} / \mathrm{L}$ ) y la cavidad oral se irradió con un LED azul $(468 \mathrm{~nm})$

\section{Carta al Editor}

\author{
Sandra Martínez-Pizarro 1,a \\ ${ }^{1}$ Hospital comarcal de Huércal Overa, Almería, España. \\ a Enfermera.
}

\section{Correspondencia:}

Sandra Martínez-Pizarro: mpsandrita@hotmail.com Avd Murcia km 175 frente hotel Robemar. 18800 Baza Granada. España.

ORCID: 0000-0003-3070-8299

\section{Conflicto de intereses: ninguno.}

\section{Recibido: 15/04/20}

Aprobado: 19/04/20

Publicado: 04/08/20

(C) Los autores. Este artículo es publicado por la revista Odontología Sanmarquina de la Facultad de Odontología, Universidad Nacional Mayor de San Marcos. Este es un artículo de acceso abierto, distribuido bajo los términos de la licencia Creative Commons Atribucion - No Comercia_Compartir Igual 4.0 Internacional. (http://creativecommons.org/licenses/by-nc-sa/4.0/) que permite el uso no comercial, distribución y reproducción en cualquier medio, siempre que la obra original sea debidamente citada. 
durante $5 \mathrm{~min}$. Se observaron reducciones significativas en el grado de OM después de la aplicación de PBM-T o PBM-T + PDT. PBM-T + PDT resultó en un tiempo más corto para la resolución de las lesiones en comparación con PBM-T solo. PBM-T, solo o combinado con PDT, puede usarse para el tratamiento de la OM. PDT + PBM-T en particular aceleró el proceso de curación de OM, reduciendo el tiempo de remisión de la lesión de 15 a 11 días.

En el estudio de Marín-Conde et al. ${ }^{4}$ realizado en 2019 se analizó la efectividad de la fotobiomodulación con terapia con láser de bajo nivel (LLLT) como procedimiento terapéutico para el tratamiento de la OM causada por radioquimioterapia en pacientes con cáncer. Se realizó un estudio experimental, prospectivo, doble ciego, aleatorizado y controlado en 26 pacientes: $11(42,3 \%)$ en el grupo de estudio y $15(57,7 \%)$ en el grupo control. Se observaron diferencias estadísticamente significativas entre los grupos desde la semana 5 de tratamiento. El 72,7\% del grupo láser mostró mucosa normal (mucositis grado 0), mientras que en el grupo control, $20,0 \%$ mostró mucositis grado 0 y $40,0 \%$ mostró mucositis grado 2 . No se encontraron diferencias significativas entre los grupos con respecto a la aplicación o uso de medicamentos durante todo el período de estudio. La evaluación de tolerancia no mostró ninguna diferencia estadísticamente significativa entre los grupos con respecto a la aparición de efectos secundarios o eventos adversos durante el ensayo. La fotobiomodulación con LLLT reduce la incidencia y la gravedad de la mucositis en pacientes tratados con radioterapia y/o quimioterapia.

En el estudio de Pinheiro et al. ${ }^{5}$ realizado en 2019 se examinó el uso de PBM-T en pacientes sometidos a quimioterapia y / o radioterapia y afectados por OM. Treinta y un pacientes fueron divididos en dos grupos. Uno recibió PBM-T ( $\mathrm{n}=17)$ solo, que consiste en irradiación láser de baja intensidad (LIL) (longitud de onda $660 \mathrm{~nm}$, modo continuo, energía puntual $4 \mathrm{~J}$, densidad de energía $142 \mathrm{~J} / \mathrm{cm} 2$, tiempo de irradiación $40 \mathrm{~s}$ por punto), una vez por semana durante 4 semanas. El otro $(\mathrm{n}=14)$ recibió una combinación de PBM-T y PDT, con curcumina como fotosensibilizador. Los pacientes recibieron instrucciones de enjuagarse la boca con $20 \mathrm{ml}$ de solución de curcumina durante 5 minutos. La cavidad oral se irradió con un diodo emisor de luz azul (potencia $1200 \mathrm{mV}$, longitud de onda $468 \mathrm{~nm}$ ) durante $5 \mathrm{~min}$. LIL se aplicó como en el grupo PBM-T. Después del tratamiento, se evaluó nuevamente la gravedad de la OM. Los resultados mostraron que tanto PBM-T solo como PBM-T + PDT produjeron reducciones significativas en el grado de OM. PDT + PBM-T resultó en un tiempo de curación significativamente más corto en comparación con PBM-T solo.

Tras examinar los resultados de los estudios expuestos anteriormente, se puede observar el potencial de la fotobiomodulación en el manejo y tratamiento de la mucositis oral. Además de su eficacia, los resultados muestran que la combinación de fotobiomodulación con terapia fotodinámica acelera el proceso de curación de OM reduciendo el tiempo de remisión de la lesión. Según la literatura, la fotobiomodulación debería usarse 4 días a la semana con una duración de 10-15 minutos; desde el primer hasta el último día de radioquimioterapia o hasta la resolución de la mucositis con una energía de 1,0 J de energía. En el caso de combinar fotobiomodulación con PDT los estudios recomiendan una aplicación por semana durante 4 semanas con una energía puntual de $4 \mathrm{~J}$ y un tiempo de irradiación de 40 segundos por punto.

Sin embargo, para establecer recomendaciones generalizadas es necesario incrementar la cantidad de ensayos clínicos con una metodología precisa, mayor número de muestra y con un estándar de calidad alto. Con ello se podrá examinar la eficacia y posibles complicaciones a corto y largo plazo, explorar su posible efecto sinérgico con otras terapias y analizar su rentabilidad económica. De esta forma, los profesionales sanitarios podrán ofrecer a los pacientes los mejores cuidados basados en las últimas evidencias científicas demostradas.

\section{Referencias bibliográficas}

1. Elad S, Arany P, Bensadoun RJ, Epstein JB, Barasch A, Raber-Durlacher J. Photobiomodulation therapy in the management of oral mucositis: search for the optimal clinical treatment parameters. Support Care Cancer. 2018;26(10):3319-3321. DOI: 10.1007/s00520-0184262-6.

2. Guedes CDCFV, de Freitas Filho SAJ, de Faria PR, Loyola AM, Sabino-Silva R, Cardoso SV. Variation of energy in photobiomodulation for the control of radiotherapy-induced oral mucositis: A clinical study in head and neck cancer patients. Int J Dent. 2018;2018:4579279. DOI: $10.1155 / 2018 / 4579279$.

3. Pires Marques EC, Piccolo Lopes F, Nascimento IC, Morelli J, Pereira MV, Machado Meiken VM, et al. Photobiomodulation and photodynamic therapy for the treatment of oral mucositis in patients with cancer. Photodiagnosis Photodyn Ther. 2020;29:101621. DOI: 10.1016/j.pdpdt.2019.101621.

4. Marín-Conde F, Castellanos-Cosano L, Pachón-Ibañez J, Serrera-Figallo MA, Gutiérrez-Pérez JL, Torres-Lagares D. Photobiomodulation with low-level laser therapy reduces oral mucositis caused by head and neck radio-chemotherapy: prospective randomized controlled trial. Int J Oral Maxillofac Surg. 2019;48(7):917-923. DOI: 10.1016/j.ijom.2018.12.006.

5. Pinheiro SL, Bonadiman AC, Borges Lemos ALDA, Annicchino BM, Segatti B, Pucca DS, et al. Photobiomodulation therapy in cancer patients with mucositis: A clinical evaluation. Photobiomodul Photomed Laser Surg. 2019;37(3):142-150. DOI: 10.1089/photob.2018.4526. 